\title{
Efficient Communication and Collection with Compact Normal Forms
}

\author{
Edward Z. Yang ${ }^{1} \quad$ Giovanni Campagna $^{1} \quad$ Ömer S. Ağacan ${ }^{2} \quad$ Ahmed El-Hassany $^{2}$ \\ Abhishek Kulkarni $^{2} \quad$ Ryan R. Newton ${ }^{2}$ \\ Stanford University (USA) ${ }^{1}$, Indiana University (USA) ${ }^{2}$ \\ \{ezyang, gcampagn\}@cs.stanford.edu \{oagacan, ahassany, adkulkar, rrnewton\}@indiana.edu
}

\begin{abstract}
In distributed applications, the transmission of non-contiguous data structures is greatly slowed down by the need to serialize them into a buffer before sending. We describe Compact Normal Forms, an API that allows programmers to explicitly place immutable heap objects into regions, which can both be accessed like ordinary data as well as efficiently transmitted over the network. The process of placing objects into compact regions (essentially a copy) is faster than any serializer and can be amortized over a series of functional updates to the data structure in question. We implement this scheme in the Glasgow Haskell Compiler and show that even with the space expansion attendant with memory-oriented data structure representations, we achieve between $\times 2$ and $\times 4$ speedups on fast local networks with sufficiently large data structures.
\end{abstract}

Categories and Subject Descriptors D.3.2 [Programming Languages]: Language Classifications - Concurrent, Distributed, and Parallel Languages

Keywords Serialization, Message Passing, Regions

\section{Introduction}

In networked and distributed applications it is important to quickly transmit data structures from one node to another. However, this desire is often in tension with the usual properties of high-level languages:

- Memory-safe languages such as Haskell or Java support rich, irregular data structures occupying any number of non-contiguous heap locations.

- In contrast, network interface cards (NICs) perform best when the data to be sent resides in a single contiguous memory region, ideally pinned to physical memory for direct memory access (DMA).

Thus, while efficiently sending byte arrays does not pose a problem for high-level languages, more complex data structures require a serialization step which translates the structure into a contiguous

This is the author's version of the work. It is posted here for your personal use. Not for redistribution. The definitive version was published in the following publication:

ICFP'15, August 31 - September 2, 2015, Vancouver, BC, Canada ACM. 978-1-4503-3669-7/15/08...

http://dx.doi.org/10.1145/2784731.2784735 buffer that is then sent over the network. This serialization process is a source of overhead and can be the limiting factor when an application runs over a fast network.

In response to this problem, there have been several attempts to engineer runtime support enabling high-level languages to send heap representations directly over the network: e.g. in Java [9], or even in distributed Haskell implementations [19]. However, these approaches rarely manage to achieve zero-copy data transmission, and complications abound with mutable and higher order data.

In this paper, we propose a new point in the design space: we argue it's worth adopting the same network representation as the native in-memory representation, despite the cost in portability and message size. We show that even when message size increases by a factor of four, on a fast local network-like those found in data centers or supercomputers-end-to-end performance can still be improved by a factor of two.

In effect, the problem of fast network transfer reduces to the problem of arranging for heap data to live in contiguous regions. While region type systems $[2,12,30]$ could address this problem, we implement a simpler solution which requires no changes to the type system of Haskell: let programmers explicitly place immutable data into compact regions or compact normal form (CNF). Objects in these regions are laid out in the same way as ordinary objects: they can be accessed in the same way from ordinary Haskell code and updated in the standard manner of purely functional data structures (the new nodes appended to the compact region). Furthermore, as the data in question is immutable and has no outgoing pointers, we side step the normal memory management problems associated with subdividing the heap (as in generational and distributed collectors). Finally, given any heap object we can quickly test for membership in a compact region, from which we can also deduce whether it is fully evaluated, a question which is often asked in a lazy language like Haskell.

Adding CNF to Haskell also solves two other, seemingly unrelated problems:

- Permanent data residency. In long-running programs, there may be some large data structures which never become garbage. With a standard generational garbage collector, these data structures must still be fully traversed upon a major GC, adding major overhead. In these cases, it is useful to promote such data to an immortal generation which is never traced.

- Repeated deepseq. Even setting aside serialization, there are other reasons to fully evaluate data, even in a lazy language. For example, in parallel computation settings, it is important to ensure that computational work is not accidentally offloaded onto the wrong thread by transmission of a thunk. 
This hyperstrict programming in Haskell is done with the NFData type class, which permits a function to deeply evaluate a structure to normal form. However, deepseq (deepseq $\mathrm{x}$ ) demonstrates a problem with the approach. The second deepseq should cost $O(1)$, as the data is already in normal form. However, as there is no tracking of normal forms either in the type system or the runtime, Haskell's NFData methods must perform repeated traversals of data, which can easily lead to accidental increases in the asymptotic complexity of an algorithm.

Once data is compacted in a CNF, repeated deepseq becomes $O(1)$, and the garbage collector likewise never needs to trace the data again. More generally, we make the following contributions:

- We propose a basic API for CNFs, specify what invariants it enforces and formally describe some of its sharing properties. Our default API does not preserve sharing when copying data structures into a compact region; however, at the cost of a factor of two, sharing can be preserved by tracking copied nodes in an auxiliary data structure (e.g., a hash table).

- We implement CNFs by modifying the Glasgow Haskell Compiler (GHC) and runtime and compare CNF to accepted, highperformance serialization approaches for Haskell. We demonstrate while that Haskell serialization is competitive with welloptimized alternatives (e.g. the Oracle Java virtual machine), the CNF approach is radically faster. Further, we quantify how this serialization advantage translates into faster message passing or remote procedure calls (Section 5.7), including when used in conjunction with remote direct memory access.

- We show that CNF can also improve garbage collection: both in reducing GC time and scaling to large heaps (Section 5.5). CNFs offer a middle ground that enables some application control of heap storage without compromising type safety or requiring major type-system changes.

While the specific low-level techniques applied in this paper are not novel, we hope to show that with this work, distributed functional programming can become much more efficient than it has been. This is especially apt, as in recent years there has been extensive work on distributed Haskell frameworks [10, 20], which depend on slow serialization passes to send data.

\section{Motivation: Serialization and its Discontents}

Consider the simple problem of building and then sending a tree value to another process:

sendBytes sock (serialize (buildTree $\mathrm{x}$ ))

In general, serializing the tree, that is, translating it into some welldefined format for network communication, is unavoidable, since the receiving process may be written in a completely different language, by a completely different set of people, in which case a portable interchange format is necessitated.

However, there are some situations where endpoints may be more closely related. If we are sending the tree to another thread in the same process, no serialization is necessary at all: just send the reference! Even in a distributed computation setting, it is relatively common for every process on the network to be running the same binary. We can summarize the possible situations by considering who we are sending to:

1. Another thread in the same process;

2. Another process in the network, trusted to be running the same binary;

3. A trusted endpoint in the network, which may not run the same binary; or perhaps

\section{An untrusted endpoint across the network.}

Most serialization and networking libraries are designed for the worst case-scenario 4-and thus miss out on substantial opportunities in cases 2 and 3. In Haskell, for example, the best option today is to use a binary serialization library such as binary or cereal. These libraries are very efficient examples of their kind, but by their nature they spend substantial time packing structures into an array of bytes and then unpacking them again on the other side.

Why should we care about scenarios 2 and 3 ? While scenario 4 covers general applications interacting with the Internet, these middle scenarios represent applications running inside of supercomputers and data-centers composed of many nodes. In scenario 2, and possibly scenario 3 , we can consider sending a representation that can be used immediately on the other side, without deserialization. High-performance networking hardware that provides remote direct memory access (RDMA), makes this scenario even more appealing, as it can directly place objects in remote heaps for later consumption without the involvement of remote processors. Thus, we have this principle:

PRINCIPLE 1. To minimize serialization time, in-memory representation and network representation should be the same.

Even if we are willing to accept this principle, however, there are still some difficulties.

\subsection{Problem 1: Contiguous In-Memory Representation}

By default, data allocated to the heap in a garbage collected language will not be in a contiguous region: it will be interspersed with various other temporary data. One direct solution to this problem might be to replace (serialize (buildTree $\mathrm{x}$ )) from the earlier example code with an alternate version designed to produce a contiguous version of the tree, which could be immediately consumed by sendBytes:

sendBytes chan (buildTreeToRegion $\mathrm{x}$ )

The first problem with this approach is that its anti-modular if buildTree must be changed to yield buildTreeToRegion. The producer code may be produced by a library not under the control of the programmer invoking sendBytes - thus it is unreasonable to expect that the producer code be modified to suit the consumer. Nor is it reasonable to expect a program analysis to identify buildTree as producing network-bound data, because it is impossible to determine, in general (at all allocation sites) what the ultimate destination of each value will be. Besides, most high-level languages do not have the capability to region-allocate, even if we were willing to change the producer code.

A region-based type system with sufficient polymorphism could solve the modularity problem: a function identifies what region the returned value should be allocated into. But, while there have been languages that have this capability and expose it to users [12], widely used functional and object oriented languages do not. In fact, even MLKit [30]—which implements SML using regions and region-inference-does not expose region variables and letregion to the programmer. Thus they cannot write buildTreeToRegion and cannot guarantee that the result of builtTree ends up as the sole occupant of a distinct region.

Due to these drawbacks, we instead propose much simpler scheme: to simply copy the relevant data into the contiguous region. The key principle:

PRINCIPLE 2. Copying is acceptable, as long as the copy is amortized across all sends of the same data.

In fact, when a copying garbage collector would be used, live data structures would have been copied anyway. We can do the copy 
once, and then avoid any further copies (by the garbage collector or otherwise.)

\subsection{Problem 2: Safety}

Once your data is in a contiguous, compact region, one would hope that it would simply be possible to send the entire region (without any checking) when attempting to send a pointer to an object in the region.

However, such an operation is only safe if the region, in fact, contains all of the reachable objects from a pointer. If this has been guaranteed (e.g., because a copy operates transitively on reachable objects), there is yet another hazard: if mutation is permitted on objects in the compact region, then a pointer could be mutated to point out of the region.

In fact, an analogous hazard presents itself with garbage collection: if a compact region has outbound regions, it is necessary to trace it in order to determine if it is keeping any other objects alive. However, if there are no outgoing pointers and the data is immutable, then it is impossible for a compact region to keep objects outside of it alive, and it is not necessary to trace its contents. To summarize:

PRINCIPLE 3. Immutable data with no-outgoing pointers is highly desirable, from both a network transmission and a garbage collection standpoint.

\section{Compact Normal Form}

Our goal with CNFs is to organize heap objects into regions, which can then be transmitted over the network or skipped during garbage collection. Concretely, we do this by representing a pointer to an object in a compact region with the abstract type Compact a. Given a Compact a, a pointer to the actual object can be extracted using getCompact:

newtype Compact a

getCompact : : Compact a $\rightarrow$ a

How do we create a Compact a? Based on the properties of compact regions we have described so far, any such operation would need to take a value, fully evaluate it, and copy the result into a contiguous region. We represent the types which can be evaluated and copied in this way using a type class Compactable, similar to an existing Haskell type class NFData which indicates that a type can be evaluated to normal form. Unlike NFData, Compactable expresses the added capability to send some data over the network. Most common types are compactable, e.g. Bool or Maybe a (if a is Compactable), but mutable types such as IORef a are not.

class NFData a $\Rightarrow$ Compactable a

We might then define a function with this type:

newCompact : : Compactable a $\Rightarrow \mathrm{a} \rightarrow$ IO (Compact a)

This function creates a new region and copies the fully evaluated a into it. However, if we want to apply a functional update to this tree, we may want to specify the already existing compact region so we can reuse any already compacted shared data. To do this, we can decompose newCompact into two functions:

$$
\begin{array}{ll}
\text { mkCompact } & :: \text { IO (Compact ()) } \\
\text { appendCompact } & :: \text { Compactable a } \\
& \Rightarrow \mathrm{a} \rightarrow \text { Compact } \mathrm{b} \rightarrow \text { IO (Compact a) }
\end{array}
$$

mkCompact simply creates a new region and returns a dummy pointer Compact () to identify the region. appendCompact, like newCompact, fully evaluates a; however, it copies the result into the same compact region as Compact b. Additionally, it short-circuits

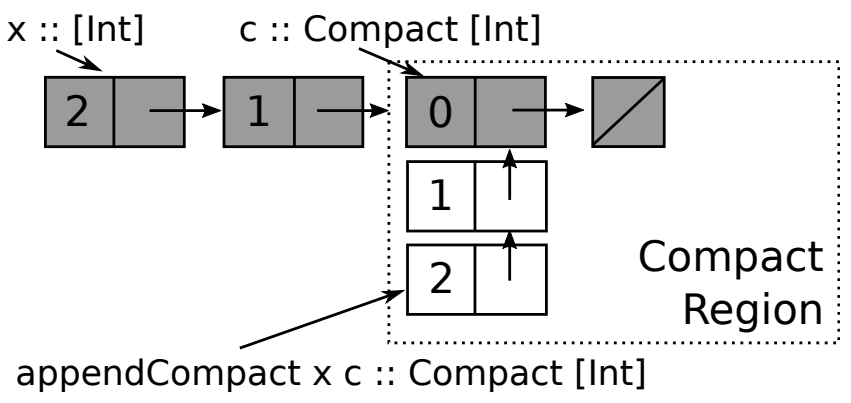

Figure 1: Appending a list of (unboxed) integers into a compact region. The white boxes are the newly allocated objects in the region after the append which share a tail with the original list.

the evaluation/copying process if a subgraph is already in the target compact region. (The actual heap object Compact b points to is otherwise ignored.) Figure 1 gives an example of appending some cells of a list to a compact region; in this example, both $a$ and $b$ are the same type-however, this need not necessarily be the case.

While one could quibble with the particular interface provided (perhaps compact regions should be distinguished from compact pointers), the above interface is sufficient for all compactions. However, beyond this core interface, one will need to provide support for sending Compact a values over the network, e.g.:

sendCompact : : Socket $\rightarrow$ Compact a $\rightarrow$ IO ()

as in this example:

do $c \leftarrow$ newCompact (buildTree $\mathrm{x}$ )

sendCompact sock c

(Un)observable sharing Interestingly, you cannot observe sharing of Haskell values with just mkCompact and appendCompact. In particular, if we ignore performance, we could implement observably equivalent pure versions of these functions in the following way (where deepseq is a method in NFData which evaluates its first argument to normal form when the second argument is forced):

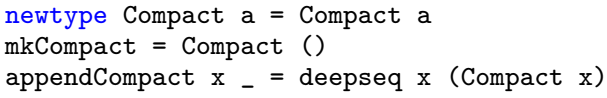

Of course, the (useful) function which tests if an arbitrary value lives in a compact region does permit observing the presence of sharing:

isCompact $::$ a $\rightarrow$ IO (Maybe (Compact a))

\subsection{Region Invariants}

The Compactable type class enforces some important safety invariants on the data which lives in a compact region:

- No outgoing pointers. Objects are copied completely into the compact region, so there are never any outgoing pointers. This is useful when transmitting a region over the network, as we know that if we send an entire region, it is self-contained. We will also rely on this invariant in garbage collection (described in more detail in Section 4): this invariant means it is not necessary to trace the inside of a region to determine liveness of other objects on the heap. Compacted objects are essentially a single array-of-bits heap object.

- Immutability. No mutable objects are permitted to be put in a compact region. This helps enforce the invariant of no outgoing pointers, and also means that data in a region can be copied with impunity. 
- No thunks. Thunks are evaluated prior to being copied into a region; this means the CNF will not change layout, be mutated, or expand as a result of accessing its contents, and that we do not attempt to send closures over the network.

Haskell has especially good support for immutable data, which makes these restrictions reasonable for compact regions. While many languages now host libraries of purely functional, persistent data structures, in Haskell these are used heavily in virtually every program, and we can reasonably expect most structures will be Compactable.

\subsection{Sharing}

Because every compact region represents a contiguous region of objects, any given object can only belong to at most one compact region. This constraint has implications on the sharing behavior of this interface. Here are three examples which highlight this situation:

Sharing already compact subgraphs Consider this program:

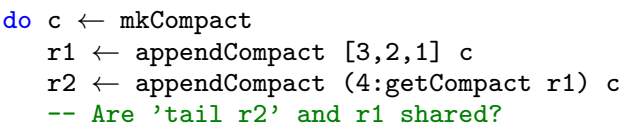

In the second appendCompact, we are adding the list $[4,3,2,1]$. However, the sublist $[3,2,1]$ is already in the same compact region: thus, it can be shared.

However, suppose $r 1$ is in a different compact, as here:

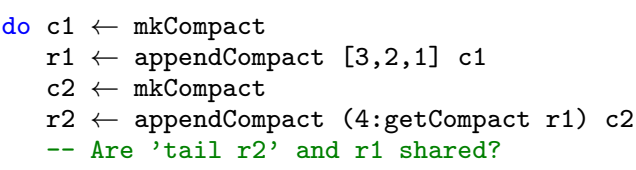

In this case, sharing would violate the compact region invariant. Instead, we must recopy $r 1$ into the new compact region. The copying behavior here makes it clear why, semantically, it doesn't make sense to allow mutable data in compact regions.

Sharing non-compact subgraphs We stated that if if a subgraph is already compact, it can be shared. What if the subgraph is not compact?

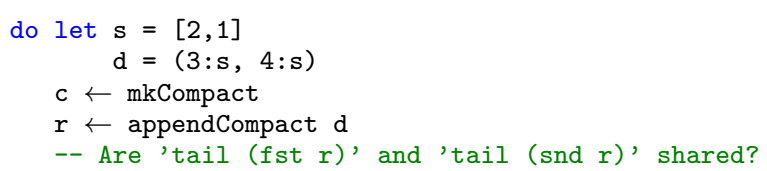

In an ideal world, the sharing present in d would be preserved in the compact region. However, for reasons we will describe in Section 4.3, we can more efficiently implement copying if we don't preserve sharing. Thus, by default, we do not preserve sharing of non-compact subgraphs; however, a user may optionally use a slower API to preserve sharing.

Sharing after append Consider the following program, where $t$ is a thunk whose type is compactable:

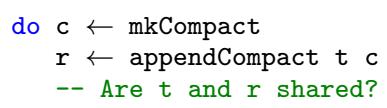

The process of appending $t$ to c caused it to be fully evaluated; furthermore, $r$ refers to the fully evaluated version of this data structure which lives in the compact region. Is $t$ updated to also point to this data structure?

In some cases, it is not possible to achieve this sharing: if $t$ is a reference to a fully evaluated structure in different compact, it must

$\begin{array}{ll}e \quad:= & \\ \mid \begin{array}{l}\text { lit } \\ f \overline{a_{i}}\end{array} & \text { Literal } \\ x & \text { Application } \\ K \bar{a}_{i} & \text { Variable } \\ \text { case } e \text { of } \overline{K_{i} \bar{a} \rightarrow e_{i}} \mathbf{i} & \text { Constructor } \\ \text { let } x=r h s \text { in } e & \text { Pattern match } \\ \text { mkCompact } & \text { Let binding } \\ \text { appendCompact } x y & \end{array}$

$\begin{array}{ccc}\text { rhs } & := & \text { Right-hand sides } \\ & \lambda \overline{x_{i}}{ }^{i} \cdot e & \text { Function } \\ & \ulcorner e\urcorner & \text { Thunk } \\ & K \overline{a_{i}}{ }^{i} & \text { Constructor }\end{array}$

Figure 2: Syntax for simplified STG

be copied to the new compact region. Additionally, if $t$ had already been fully evaluated, it's not possible to "modify" the result to point to the version in the new compact region. Thus, to make sharing behavior more predictable and indifferent to evaluation order, we decided $t$ should never be updated to point to the version of the data structure in the compact.

Semantics We can be completely precise about the sharing properties of this interface by describing a big-step semantics for our combinators in the style of Launchbury's natural semantics [18]. To keep things concrete, we work with the specific intermediate language used by GHC called STG [15], which also supports data constructors. The syntax STG plus our combinators is described in Figure 2, with metavariables $f$ and $x$ representing variables, $K$ representing constructors, and $a$ representing either a literal or variable. STG is an untyped lambda calculus which has the same restriction as Launchbury natural semantics that all arguments $a$ to function (and constructor) applications must either be a literal or a variable. This makes it easy to model the heap as a graph (with variables representing pointers); thus, sharing behavior can be described.

The basic transition in a big-step semantics is $\Gamma: e \Downarrow \Gamma^{\prime}: a$ : an expression $e$ with heap $\Gamma$ reduces to a value or literal with new heap $\Gamma^{\prime}$. The semantics for the standard constructs in STG are completely standard, so we omit them; however, there is one important difference about $\Gamma$ : a heap may also contain labelled bindings $x \stackrel{c}{\mapsto} v$, indicating the value in question lives in a compact region $c$. (Values in the normal heap implicitly have a special label $\epsilon)$. With this addition, the rules for the two combinators are then quite simple:

$$
\begin{aligned}
& \frac{c \text { fresh } \quad x \text { fresh }}{\Gamma: \text { mkCompact } \Downarrow \Gamma[x \stackrel{c}{\mapsto}()]: x} \\
& \frac{\Gamma: x \quad \Downarrow \Delta: x^{\prime} \quad x^{\prime} \stackrel{c}{\mapsto} r h s \text { in } \Delta \quad \Delta: y \quad \Downarrow_{c}^{\text {rnf }} \Theta: y^{\prime}}{\Gamma: \text { appendCompact } x y \Downarrow \Theta: y^{\prime}}
\end{aligned}
$$

The rule for appendCompact hides some complexity, as it needs to recursively evaluate a data structure to normal form. We can express this process with a specialized evaluation rule $\Gamma: e \Downarrow_{c}^{\mathrm{rnf}}$ $\Gamma^{\prime}: a$, which indicates $e$ should be fully evaluated and the result copied into the compact region $c$, where $a$ points to the root of the copied result. The "reduce to normal form" operation ( $r n f)$ has only three rules: 


$$
\begin{aligned}
& \frac{\Gamma: e \Downarrow \Gamma^{\prime}: z^{\prime} \quad \Gamma^{\prime}: z^{\prime} \Downarrow_{c}^{\mathrm{rnf}} \Gamma^{\prime \prime}: z^{\prime \prime}}{\Gamma: e \Downarrow_{c}^{\mathrm{rnf}} \Gamma^{\prime \prime}: z^{\prime \prime}} \text { EVAL } \\
& \frac{x \stackrel{c}{\mapsto} v \text { in } \Gamma}{\Gamma: x \Downarrow_{c}^{\text {rnf }} \Gamma: x} \quad \text { SHORTCUT }
\end{aligned}
$$

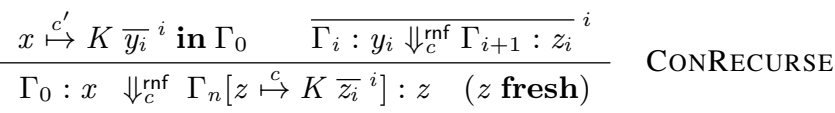

First, if data pointed to by $x$ is not fully evaluated, we evaluate it first using the standard reduction rules (EvAL). Otherwise, if we are attempting to rnf a variable into $c$ (SHORTCUT), but it already lives in that region, then nothing more is to be done. Otherwise, $x$ already points to a constructor in weak head normal form but in a different region $c^{\prime}$ (CONRECURSE), so we recursively rnf the arguments to the constructor, and then allocate the constructor into the compact region $c$.

It is easy to show by induction that compact region invariant is preserved by these evaluation rules:

INVARIANT 1 (Compact region invariant). For any heap binding $x \stackrel{c}{\mapsto} v$ in $\Gamma$ where $c$ is not $\epsilon$, $v$ is a constructor $K{\overline{a_{i}}}^{i}$ such that for each non-literal variable $a_{i}, a_{i} \stackrel{c}{\mapsto} v_{i}$ is in $\Gamma$.

THEOREM 1 (Preservation). If the compact invariant holds on $\Gamma$, and $\Gamma: e \Downarrow \Gamma^{\prime}: z^{\prime}$, then the compact invariant holds on $\Gamma^{\prime}$.

\section{Implementation}

\subsection{The GHC Runtime System}

We first review some details of GHC runtime system. Readers already familiar with GHC's internals can safely skip to the next subsection.

Block-structured heap In GHC, the heap is divided in blocks of contiguous memory in multiples of $4 \mathrm{~KB}$. [21] The smallest block size is $4 \mathrm{~KB}$, but larger blocks can be allocated to hold objects which are larger than $4 \mathrm{~KB}$. Blocks are chained together in order to form regions of the heap, e.g. the generations associated with generational garbage collection.

In memory, blocks are part of aligned megablocks of one megabyte in size. These megablocks are the unit of allocation from the OS, and the first few blocks in each megablock are reserved for the block descriptors, fixed size structures containing metadata for one block in the same megablock. Because of this organization it is possible to switch between a block and a block descriptor using simple pointer arithmetic. Block descriptors contain information such as how large a block is (in case it holds an object larger than four kilobytes) and what portion of the block is in use.

This block structure gives the GHC runtime system the property that given an arbitrary pointer into the heap, it is possible in some cases to verify in constant time in what object it lives, and that property is exploited by our implementation to efficiently test if an object already lives in a compact region.

Layout of objects in the heap Since the in-memory representation of objects is what will be transmitted on the network, it is worth explaining how GHC lays out objects in memory. Objects are represented by a machine size info pointer followed by the payload of the object (numeric data and pointers to other object, in an order which depends on the object type).

The info pointer points to an info table, a static piece of data and code that uniquely identifies the representation of the object and the GC layout. In case of functions, thunks and stack continuations, it holds also the actual executable code, while for ADTs it contains an identifier for the constructor which is used to discriminate different objects in case expressions.

It is important to note that info tables are stored along side the machine code in the executable and never change or move for the lifetime of the process. Moreover, in case of static linking, or dynamic linking without address space layout randomization, they are also consistent between different runs of the same binary. This means that no adjustment to info pointers is necessary when the same binary is used.

\subsection{Compact Regions}

Conceptually, a compact region is a mutable object in which other objects can be added using the appendCompact operation. Operationally, a region is represented as a chain of blocks (hopefully one block long!) Each block of a compact region has a metadata header (in addition to the block descriptor associated with the block), which contains a pointer to the next and to the first block in the chain. Additionally, the first block of a compact region contains a tag which identifies the machine from which the data originated (the purpose of which is explained later in the section).

It is interesting to observe therefore that a compact region can be thought of as a heap object in itself: it can be treated as a linked list of opaque bytestrings which do not have to be traced. At the same time, the compact region internally contains other objects which can be directly addressed from outside.

Garbage collection Usually in a garbage collected language, it is unsafe to address component parts of an object known to the GC, because there is no way for the GC to identify the container of the component and mark it as reachable as well.

Nevertheless, for compacts this property is achievable: given an arbitrary address in the heap, we can find the associated block descriptor and use the information stored there to verify in constant time if the pointer refers to an object in a compact storage. If it does, we mark the entirety of the compact region as alive, and don't bother tracing its contents. This test can be used by user code to check if an object is already a member of a compact, or even if it is just in normal form (so a deepseq can be omitted).

Skipping tracing of the insides of compact regions has one implication: if a single object in a compact region is live, all objects in a compact region are live. This approximation can result in wasted space, as objects which become dead cannot be reclaimed. However, there a few major benefits to this approximation. First, longlived data structures can be placed in a compact region to exclude them from garbage collection. Second, the avoidance of garbage collection means that, even in a system with copying garbage collection, the heap addresses of objects in the region are stable and do not change. Thus, compact regions serve as an alternate way of providing FFI access to Haskell objects. Finally, a "garbage collection" can be requested simply by copying the data into a new compact region, in the same manner a copying garbage collector proceeds.

\subsection{Appending Data to a Compact Region}

As we've described, the process of appending data to a compact region is essentially a copy, short-circuiting when we encounter data which already lives in the compact region. However, we can avoid needing to perform this copy recursively by applying the same trick as in Cheney copying collection: the compact region also serves as the queue of pending objects which must be scanned and copied.

If copying would cause the block to overflow, a new block is allocated and appended to the chain, and copying then proceeds in the next block. The size of the appended block is a tunable 
parameter; in our current implementation, it is the same size as the previous block.

Preserving sharing while copying Suppose that we are copying a list into a compact region, where every element of the list points to the same object: the elements are all shared. In a normal copying garbage collector, the first time the object is copied to the new region, the original location would be replaced with a forwarding pointer, which indicates that the object had already been copied to some location.

However, in our case, we can't apply this trick, because there may be other threads of execution running with access to the original object. Initially, we attempted to preserve sharing in this situation by using a hash table, tracking the association of old objects and their copies in the compact region. Unfortunately, this incurred a significant slow-down (between $\times 1.5$ and $\times 2$ ).

Thus, our default implementation does not preserve sharing for objects which are not already in a compact region. (Indeed, this fact is implied by the semantics we have given.) Thanks to the fact that only immutable data is copied in this way, this duplication is semantically invisible to the application code, although memory requirements can in the worst case become exponential, and structures containing cycles cannot be handled by this API.

While it may seem like this is a major problem, we can still preserve sharing for data structures whose shared components already live in a compact region. In this case, when we take a data structure already in a compact region, apply some functional update to it, and append the result to it, the shared components of the new data structure continue to be shared. We believe internal sharing which does not arise from this process is less common, especially in data which is to be sent over the network.

Trusted Compactable instances The Compactable type class serves two purposes: first, it describes how to evaluate data to normal form while short-circuiting data which is already in normal form (the existing type class NFData always traverses the entirety of an object), and second, it enforces the safety invariant that no mutable objects be placed in a compact region.

Unfortunately, because Compactable type classes are user definable, a bad instance could lead in the type checker accepting a copy of an impermissible type. Currently, our implementation additionally does a runtime check to ensure the object fulfills the invariants. Ideally, however, a Compactable would only be furnished via trusted instances provided by GHC, in a similar fashion to the existing Typeable. [17]

\subsection{Network Communication}

Once your data is in a compact region, you can use any standard techniques for sending buffers over the network. However, there are some complications, especially regarding pointers which are in the compact region, so for the sake of explicitness (and to help explain the experimental setups in the next section), we describe the details here.

Serialization A compact region is simply a list of blocks: thus, the serialization of a compact region is each block (and its length), as well as a pointer to the root of the data structure that is the root. The API we provide is agnostic to the particular network transport to be used:

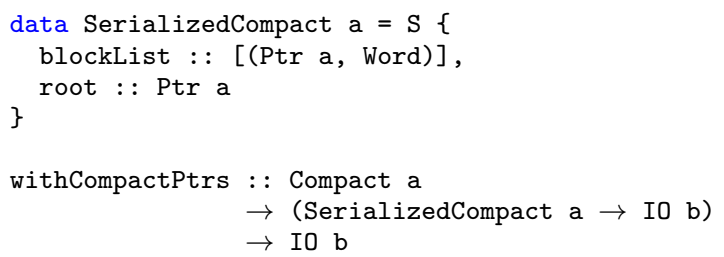

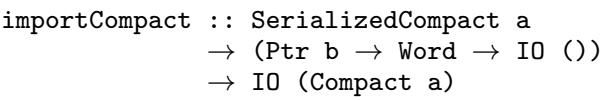

The two functions operate in pair: withCompactPtrs accepts a function SerializedCompact $\mathrm{a} \rightarrow \mathrm{IO}$ b that should write the data described by the SerializedCompact to the communication channel. Conversely, importCompact takes care of reserving space in the heap for the compact region using the SerializedCompact (transmitted out of band as simple address/size pairs), then calls the provided function Ptr $\mathrm{b} \rightarrow$ Word $\rightarrow$ IO () for each piece of reserved space: this function receives the data and places it at this address.

One property of this design is that the SerializedCompact, containing the addresses of blocks on the originating machine, must be sent in full through an out of band channel. This is to give a chance to the runtime system to allocate the blocks on the receiving machine at the right addresses from the start, which is necessary to allow full zero-copy transfer in a RDMA scenario.

Pointer adjustment If the data associated with a compact region is not loaded into the same address as its original address, it is necessary to offset all of the internal pointers so that they point to the new address of the data in question. This procedure can be skipped if the sender is trusted and the compact region is loaded to its original address.

To ensure that we will be able to load compact regions into the correct address space, we observe the address space in a 64bit architecture (or even a 48 bit one like x86_64) is fairly large, more than the application will need. Therefore, our approach is to divide it into $n$ chunks (in our case, 256 chunks of $128 \mathrm{GiB}$ each) and assign each chunk to a specific machine/process combination.

Memory in these chunks is separated by the normal heap and is used only for compact storage, which means that every machine can have an approximate view of the contents of its assigned chunk in all other machines. This is enough to greatly reduce the number of collisions when attempting a directed allocation.

Unfortunately, this scheme is not guaranteed to work, as memory can be reused on the sender before it is reclaimed also on the receiver, triggering a collision and a linear pointer adjustment. An alternate design is to never reuse address space, simply unmapping the address for old compacts when they become no longer reachable.

Interoperation with different binaries As mentioned above, info tables for each object in a compact region are static and welldefined for a given binary. This allows us to ignore the info pointers inside the compact data, provided that the data originates from another instance of the same executable on a compatible machine. We verify this with an MD5 checksum of the binary and all loaded shared libraries, which is included in the payload of every compact sent on the wire and verified upon importing.

If this verification fails, the import code has to adjust the info pointers of all objects contained in the imported storage. One option to reconstruct the info pointers would be to send the info tables together with the data. Unfortunately, the info tables are fairly large objects, due to alignment and the presence of executable code, which makes this option not viable in practice. Additionally, the executable code can potentially make references to other pieces of code in the runtime system.

Instead, we observed that every info table is identified by a dynamic linker symbol which is accessible to the runtime. Thus, we extended the compact storage format to contain a map from all info table addresses to the symbol names, to be sent on the wire with the data. This map is employed to obtain symbol names for the transmitted info table pointers, which can then be linked against their true locations in the new binary. 


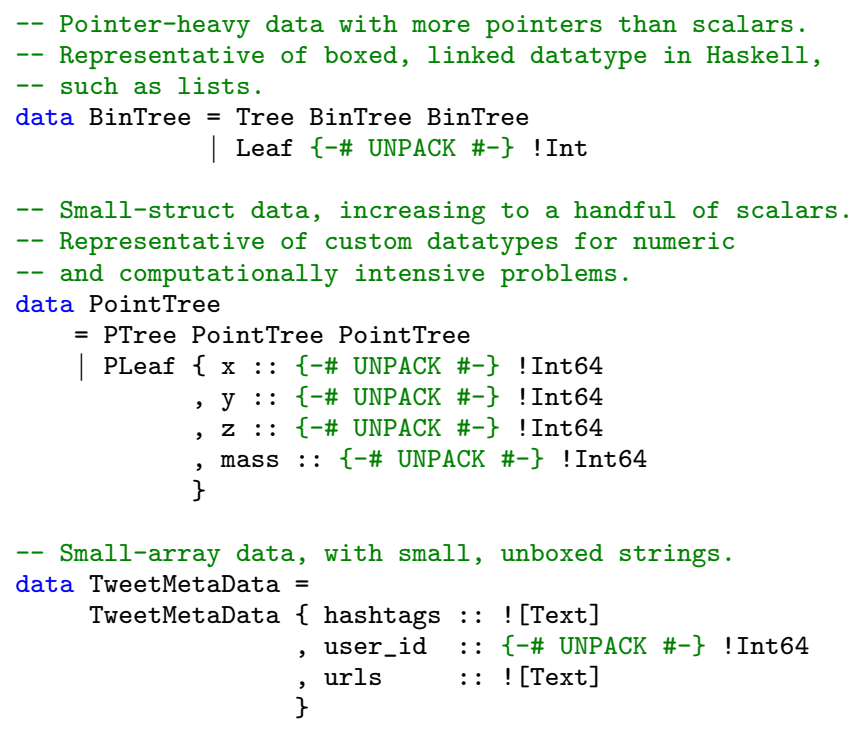

Figure 3: Our three representative data types for studying data transfer and storage. We do not cover large unboxed array data, because these types are already handled well by existing memory management and network transfer strategies.

Because this mapping incurs some overhead, we allow programmers to chose whether or not to pay this cost for more safety. On the other hand, we can cache the mapping on the receiver side, so if the types and data constructors of the values sent do not change, the setup cost needs to be paid only for the first message sent.

\section{Evaluation}

In this section, we characterize the performance of compact normal forms by looking both at serialization and memory footprint costs, as well as end-to-end numbers involving network transmission, garbage collections and a key-value store case-study. The details of the experiments are in the upcoming subsections, but we first spend some time to describe our experimental setup.

We compare against the latest versions of the Haskell binary (which operates on lazy bytestring streams) and cereal (which operates on fully evaluated bytestrings). We also compared against the builtin Java serialization engine (java.io.Serializable) shipped with Java HotSpot version 1.8.0_31, as a sanity check to ensure Haskell has reasonable performance to start with-we are not merely making slow programs less slow, nor are we addressing a Haskell specific problem.

There are a variety of different types which we could serialize and deserialize. In our experiments, we used two variants of balanced binary trees with different pointer/total size ratios, varying sizes in power of two. In particular:

- bintree is a binary tree with a single unboxed integer in leaves. This variant has high pointer/total size ratio, and thus represents a worst case scenario for transmitting compact normal forms.

- pointtree is a binary tree with four unboxed integers in leaves, increasing the data density.

Additionally, we also analyzed a third data type, composed of URLs, hashtags and user IDs for all posts in Twitter in the month of November 2012 [22, 23].

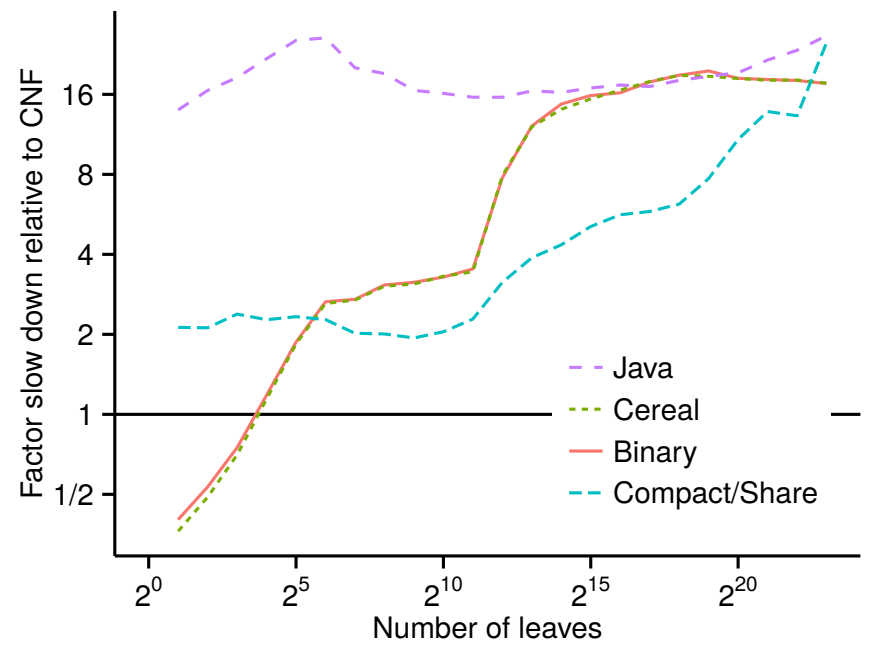

Figure 4: Relative improvement for serializing a bintree of size $2^{N}$ with CNFs versus other methods. Both $x$ and $y$ scales are logarithmic; bigger is better for CNF (and worse for the serializer being compared.) Compact/Share refers to the implementation of compact regions which preserves internal sharing, showing the overhead of the hash table.

Our experiments were done on a 16-node Dell PowerEdge R720 cluster. Each node is equipped with two 2.6GHz Intel Xeon E5-2670 processors with 8-cores each (16 cores in total), and $32 \mathrm{~GB}$ memory each. For the network benchmarks over sockets, we used the $10 \mathrm{G}$ Ethernet network connected to a Dell PowerConnect 8024F switch. Nodes run Ubuntu Linux 12.04.5 with kernel version 3.2.0.

\subsection{Serialization Costs}

Our first evaluation compares the cost of serializing data into a region, as well as the resulting space usage of the serialized versions. We don't include deserialization in this benchmark, because deserialization costs can often be pipelined with network transmission, making serialization a more representative quantity to measure. However, deserialization does add some overhead, which will be measured in the end-to-end latency benchmarks in the next section.

In Figure 4, we see a plot comparing serialization times for binary trees which store an integer at each node; some absolute values are also shown in Table ??. We can see that for sizes up to $2^{6}$, constant factors dominate the creation of compact normal forms (it takes about $1.5 \mathrm{~ns}$ to create a compact region); however, at larger sizes copying is four times faster than serializing. Beyond $2^{12}$ leaves, binary and cereal slow down a factor of four due to garbage collection overhead; by increasing the amount of memory available to GHC, this slowdown can be reduced but not eliminated.

The graph for pointtree was comparable, and for Twitter the serialization overhead was consistently $\times 5$ for binary and between $\times 4$ and $\times 9$ for Java.

\subsection{Memory Overhead}

In Table 2, we report the sizes of the various serialized representations of large versions of our data types; these ratios are representative of the asymptotic difference.

We see that in the worst case, the native in-memory representation can represent a $\times 4$ space blow-up. This is because a serialization usually elides pointers by inlining data into the stream; furthermore tags for values are encoded in bytes rather than words. 
Table 1: Median latency for serialization with CNFs versus serialization with Haskell binary and Java, for the bintree data structure.

\begin{tabular}{|c|c|c|c|}
\hline Size & Compact & Binary & Java \\
\hline \hline $2^{23}$ leaves & $0.322 \mathrm{~s}$ & $6.929 \mathrm{~s}$ & $12.72 \mathrm{~s}$ \\
$2^{20}$ leaves & $38.18 \mathrm{~ms}$ & $0.837 \mathrm{~s}$ & $1.222 \mathrm{~s}$ \\
$2^{17}$ leaves & $4.460 \mathrm{~ms}$ & $104.1 \mathrm{~ms}$ & $109 \mathrm{~ms}$ \\
$2^{14}$ leaves & $570 \mathrm{~ns}$ & $8.38 \mathrm{~ms}$ & $9.28 \mathrm{~ms}$ \\
$2^{11}$ leaves & $72.4 \mathrm{~ns}$ & $255 \mathrm{~ns}$ & $1.13 \mathrm{~ms}$ \\
\hline
\end{tabular}

Table 2: Serialized sizes of the selected datatypes using different methods.

\begin{tabular}{|c|c|c|c|c|}
\hline Method & Type & Value Size & MBytes & Ratio \\
\hline \hline Compact & bintree & $2^{23}$ leaves & 320 & 1.00 \\
Binary & & & 80 & 0.25 \\
Cereal & & & 80 & 0.25 \\
Java & & & 160 & 0.50 \\
\hline Compact & pointtree & $2^{23}$ leaves & 512.01 & 1.00 \\
Binary & & & 272 & 0.53 \\
Cereal & & & 272 & 0.53 \\
Java & & & 400 & 0.78 \\
\hline Compact & twitter & $1024 \mathrm{MB}$ & 3527.97 & 1.00 \\
Binary & & & 897.25 & 0.25 \\
Cereal & & & 897.25 & 0.25 \\
Java & & & 978.15 & 0.28 \\
\hline
\end{tabular}

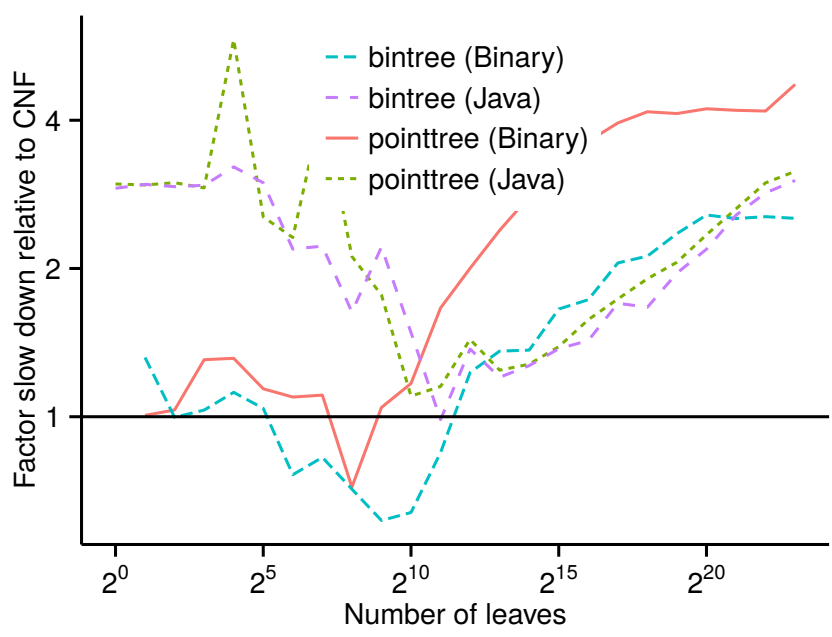

Figure 5: Relative improvement for median end-to-end latency for socket communication with CNFs versus serialization by Haskell binary and Java, for two different data structures bintree and pointtree. Both $x$ and $y$ scales are logarithmic; bigger is better for CNF (and worse for the serializer being compared.) At small sizes, constant factors of CNFs dominate.

However, as the raw data increases, our ratios do get better. Interestingly, the Twitter data achieves a relatively poor ratio: this is in part because most of the strings in this data are quite small.

The difference in memory size sets the stage for the next set of experiments on network transfer latency.

\subsection{Heap-to-Heap Network Transfer}

Given that the size of data to be transmitted increases, the real question is whether or not the end-to-end performance of transmitting a
Table 3: Median end-to-end latency for socket communication with CNFs versus serialization by Haskell binary and Java, for the different data structures bintree and pointtree.

\begin{tabular}{|c|c|c|c|c|}
\hline Type & Size & Compact & Binary & Java \\
\hline \hline bintree & $2^{23}$ leaves & $3.180 \mathrm{~s}$ & $6.98 \mathrm{~s}$ & $9.595 \mathrm{~s}$ \\
& $2^{20}$ leaves & $382.4 \mathrm{~ms}$ & $982 \mathrm{~ms}$ & $837 \mathrm{~ms}$ \\
& $2^{17}$ leaves & $59.93 \mathrm{~ms}$ & $100 \mathrm{~ms}$ & $90 \mathrm{~ms}$ \\
& $2^{14}$ leaves & $8.380 \mathrm{~ms}$ & $10.54 \mathrm{~ms}$ & $11 \mathrm{~ms}$ \\
& $2^{11}$ leaves & $1.833 \mathrm{~ms}$ & $1.238 \mathrm{~ms}$ & $2 \mathrm{~ms}$ \\
\hline pointtree & $2^{23}$ leaves & $4.978 \mathrm{~s}$ & $23.58 \mathrm{~s}$ & $15.71 \mathrm{~s}$ \\
& $2^{20}$ leaves & $624.0 \mathrm{~ms}$ & $2.64 \mathrm{~s}$ & $1.461 \mathrm{~s}$ \\
& $2^{17}$ leaves & $81.31 \mathrm{~ms}$ & $321 \mathrm{~ms}$ & $141 \mathrm{~ms}$ \\
& $2^{14}$ leaves & $13.3 \mathrm{~ms}$ & $37.1 \mathrm{~ms}$ & $35 \mathrm{~ms}$ \\
& $2^{11}$ leaves & $2.6 \mathrm{~ms}$ & $4.33 \mathrm{~ms}$ & $3 \mathrm{~ms}$ \\
\hline
\end{tabular}

heap object from one heap to another is improved by use of a compact normal form. With a fast network, we expect to have some slack: on a 1 Gbit connection, an extra 240 megabytes for a $2^{23}$ size binary tree costs us an extra 2.01 seconds; if serializing takes 6.92 seconds, we can easily make up for the slack (and things are better as more bandwidth is available).

Figure 5 shows the relative improvement for the end-to-end latency compact normal forms achieve relative to existing solutions for binary and Java. (We don't test cereal, as it does not support pipelining deserialization.) We see that for low tree sizes, constant factors and the overall round trip time of the network dominate; however, as data gets larger serialization cost dominates and our network performance improves.

\subsection{Persistence: Memory-Mapped Files}

While communicating messages between machines is the main use case we've discussed, it's also important to send messages through time, rather than space, by writing them to disk. In particular, not all on-disk storage is meant for archival purposes-sometimes it is transient, for caching purposes or communicating data between phases of an application. In Map-Reduce jobs, data is written out between rounds. Or in rendering pipelines used by movie studies, all geometry and character data is generated and written to disk from an earlier phase of the pipeline, and then repeatedly shaded in a later stage of the pipeline. For these use cases, storing in Compact format directly on disk is a feasible alternative.

Here we consider a scenario where we want to process the twitter data set discussed previously. The original data-set is stored on-disk in JSON format, so the natural way to process it would be to read that JSON. For this purpose, the standard approach in Haskell would use the efficient Aeson library ${ }^{1}$. We use Data.Aeson. TH to derive instances which parse the on-disk format to the in-memory format shown in Figure 3.

The first scenario we consider requires reading full dataset through memory, in particular we count how many occurrences of the "cat" hashtag occur in the dataset, while we vary the size of the dataset read from 1MB to 1024MB. "Aeson/all" in Figure 6 shows the result. Reading the full gigabyte takes substantial time55 seconds. "Compact/all" shows an alternative strategy. We cache a Compact representation on disk, using a format where each block is a separate file. We can then mmap these blocks directly into RAM upon loading, and allow the OS to perform demand paging

\footnotetext{
${ }^{1}$ https://hackage.haskell.org/package/aeson
} 


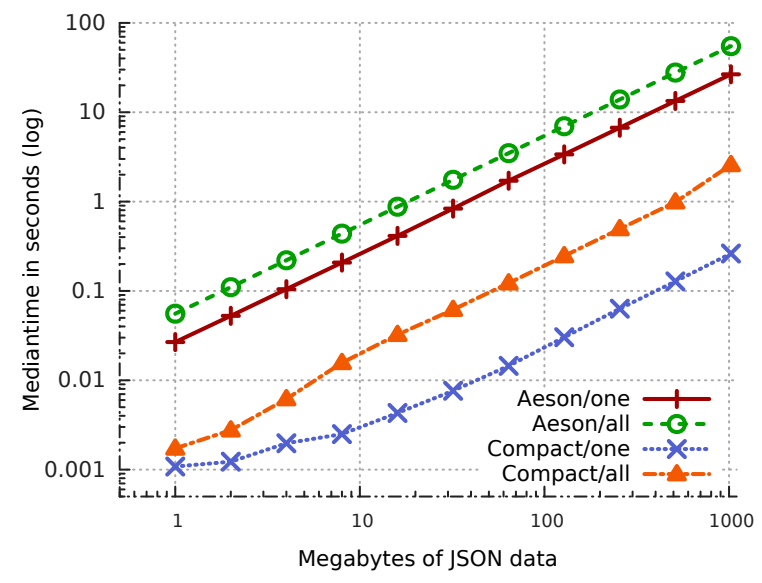

Figure 6: Time spent to load $N$ megabytes of Twitter metadata to access respectively one item at random or process all items sequentially, when loading the JSON directly with Aeson compared to loading a preprocessed Compact file from disk.

whenever we access the data. At the full $1 \mathrm{~GB}$ size, this approach is $21.3 \times$ faster than using Aeson to load the data. ${ }^{2}$

Finally, we also consider a sparse data access strategy. What if we want to read a specific tweet from the middle of the data set? This scenario measured in the "/one" variants of Figure 6. Here, we still map the entire Compact into memory. But the OS only needs to load data for the specific segments we access, no matter where they fall. As a result Compact/one still increases linearly (time for system calls to map $O(N)$ blocks), but the gap widens substantially between it and Aeson/one. The traditional parsing approach must parse half of the data set to reach the middle, resulting in 26.6 seconds to access a tweet in the middle of the 1GB dataset, rather than 0.26 seconds for Compact.

\subsection{Garbage Collection Performance}

One of the stated benefits of compact normal forms is that objects in a compact region do not have to be traced. Unfortunately, we cannot in general give an expected wall clock improvement, since the specific benefit in an application depends on what data is converted to live in a compact region. Additionally, not all data is suitable for placement in a compact region: if a data structure is rapidly changing compact regions will waste a lot of memory storing dead data.

To give a sense of what kinds of improvements you might see, we constructed a few synthetic benchmarks based on patterns we've seen in workloads where garbage collector performance is influential:

- $p$ threads concurrently allocate a list of elements into a compact region. This is a baseline showing the best-case improvement, since no elements become dead when a new cell is consed onto a list.

- $p$ threads concurrently allocate a list of elements, but rooted in a single vector. This is meant to illustrate an example where adding a compact region could help a lot, since GHC's existing parallel garbage collector scales poorly when the initial roots are not distributed across threads.

\footnotetext{
${ }^{2}$ We were not able to disable disk-caching on the evaluation platform (requiring root access), but we report the median result of 5 trials for all data points.
}

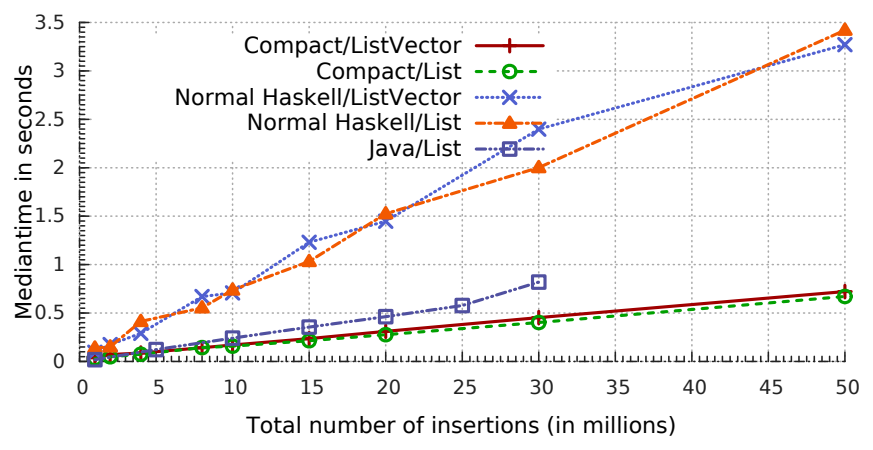

Figure 7: Median time for 16 threads to complete each $N / 16$ insertions in 16 lists, where the lists are owned by the threads separately or are referenced by a shared vector (IORef [a]). We can see that in normal Haskell times are influenced by GC pauses, which are greatly reduced for Compacts, despite the need to copy upfront. Java is included as a comparison, to show that Compact can improve performance even against a well tuned fast parallel GC.

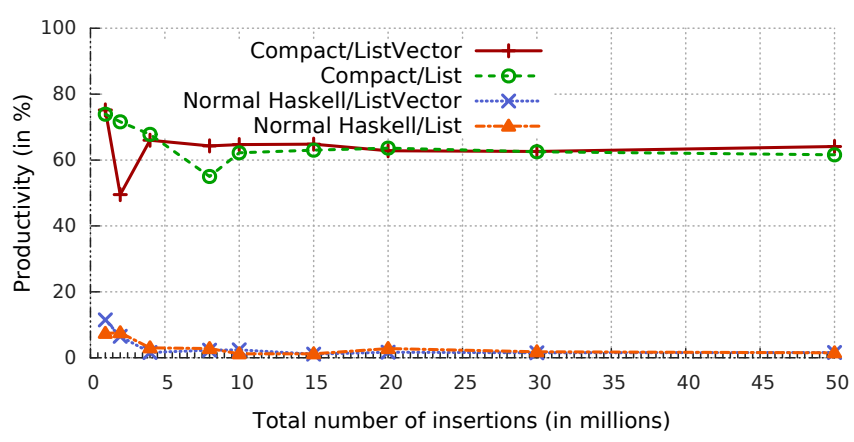

Figure 8: Percentage of CPU time spent in the mutator (as opposed to GC) for 16 threads to complete each $N / 16$ insertions in 16 lists, showing the increasing effect of tracing long lived data. Despite using a generational algorithm, the effect of major GCs is so prominent in normal Haskell that only a small fraction of time is spent in the real computation.

In all of these experiments, the data allocated by each thread is kept live until the end of the test run, simulating immortal data which is allocated but never freed.

In Figure 7 we can see the improvement in median running time for these two experiments when the operations happen for a list that lives in a compact region as opposed to the normal heap, while in Figure 8 we can observe the influence of GC in the overall time, which is greatly reduced in the compact case, allowing a more efficient use of resources.

One observation from these experiments is that it is important that the most or all of the existing compact data structure is reused by the mutator - otherwise, the excessive copies into the compact region of soon to be unused data become predominant in the total cost.

Additionally, because copying into Compact counts as allocation, this double allocation factor introduces memory pressure that triggers more garbage collections: while GC is faster in presence of compact regions, minor collections have to trace the new temporary objects that are allocated prior to copying into the compact region, and that is an added cost if the objects are short lived. 
One way to overcome this limitation is to copy the data into a new compact region after a certain number of updates, just like a copying GC would do, such that the amount of unused values is always limited. In our current implementation this is a manual process and relies on the programmer to know the space complexity of the data structure being updated as well as the access patterns from the application (possibly with the help of profiling), but future work could explore efficient heuristics to automate this.

Conversely, it may be interesting to observe that because the GC does not trace the internals of compacts, the GC pauses are less dependent on the layout of the data in memory and how it was computed, making them not only shorter but also more predictable for environments with latency constraints.

\subsection{Zero-copy Network Transfer using RDMA}

High-performance computing environments-as well as large data centers - typically are comprised of tightly-coupled machines networked using low-latency, high-throughput, switched fabrics such as Infiniband or high-speed Ethernet. Remote Direct Memory Access (RDMA) enables a source machine to remotely access a destination machine's memory without any active participation from the latter. In essence, RDMA decouples data movement from synchronization in communication between hosts. RDMA-enabled network hardware is set up to access a remote processor's memory without involving the operating system on either end. This eliminates synchronization overheads and multiple redundant copies, achieving the lowest possible latency for data movement.

The promise of fast, low-latency RDMA communication, however, is often thwarted by pragmatic issues such as explicit buffer management and synchronization, and the fact that RDMA APIs are low-level and verbose to program with. In contemporary RDMA networking hardware, a host application is required to pin the memory that it wants to expose for transfers. The operating system populates page table entries (PTE) associated with this pinned buffer such that all subsequent accesses to memory bypass the OS (the Network Interface Card (NIC) can directly DMA to or from the locked memory). Further, a source machine requires a handle to the remote memory that it wants to access. Thus, there is often a rendezvous required between peers before they can communicate with each other.

Modern high-performance communication libraries offer several features built on top of the raw RDMA API to ease message passing over the network. Each peer reserves pre-pinned ring buffers for every other peer, which are used for transferring small messages. A peer maintains an approximate pointer into a eager ring buffer which is used as an index into remote memory. When a peer suspects that it might overflow the remote buffer, it reclaims space by synchronizing with the remote peer. Large messages are sent by sending a handle to the memory, and requesting the target to get the memory associated with the handle. In addition to raw remote memory access (RMA), message passing libraries also provide a RPC mechanism for invoking handlers on the transferred remote data.

We have already discussed the interaction of CNFs with network communication, and demonstrated the claimed performance improvements in Section 5.3. Here we consider true zero-copy transfer of heap objects between two networked machines. The two cases that we evaluated are shown in Figures $9 \mathrm{a}$ and $9 \mathrm{~b}$.

Consider a case where a client wants to send a pointer-based data structure to the server. With RDMA, the client needs to know where to put the data in the server's memory. In the approach demonstrated in Figure 9a that we refer to as the eager (pushbased) protocol, the server sends a handle to a pinned region in its memory per a client's request. The client has to serialize the data structure into a contiguous memory region if the structure is not in

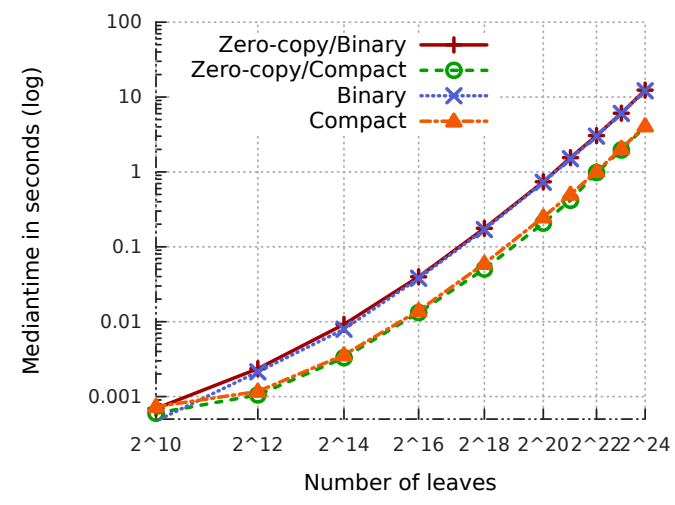

Figure 10: Median time it takes to send a bintree of varying tree depths from a client to the server using RDMA. At depth=26, it takes $48 \mathrm{~s}$ to serialize, send and deserialize a $640 \mathrm{MB}$ Binary tree (for a throughput of $13 \mathrm{MB} / \mathrm{s}$ ), whereas it takes $16 \mathrm{~s}$ for a $2.5 \mathrm{~GB}$ Compact tree (for a throughput of $160 \mathrm{MB} / \mathrm{s}$ ).

CNF. The client puts into remote memory and notifies the server of completion. All of the protocol messages are exchanged over a control channel also implemented on top of RDMA using eager ring buffers. Finally, the server deserializes the received structure incurring an extra copy and the penalty of fixing up internal pointers if the structure is in CNF.

In the rendezvous (pull-based) zero-copy case shown in Figure $9 \mathrm{~b}$, both client and server applications use the striped allocation scheme described earlier. The client has a fixed symmetric memory region (stripe) corresponding to the client in it's virtual address space. The client sends the metadata of the structure (pointer to all of the blocks) that it wants to send to the server. In the normal case, this would mean pinning each node in the tree and sending its address to the server. Fortunately, for us, a Compact is internally represented as a list of large blocks, and thus incurs significantly lower metadata exchange overhead. The server finally gets all of the blocks directly into the right addresses eliminating the need for any extraneous copies. Essentially, with this scheme, we turn all of the RDMA puts into gets, and eliminate an additional round-trip between the client and server.

The RDMA benchmarks were run over the 40Gbps QDR Infiniband interconnect through a Mellanox ConnectX-3 EN HCA. For these experiments, we used the Common Communication Interface $(\mathrm{CCI})^{3}$ RDMA library over Infiniband. We varied the depth of the tree and its data type as in the previous sections. Both protocols discussed above were implemented and the median time of each phase: tree generation, serialization, communication, deserialization was measured. At higher tree depths, the metadata for each tree is several MBs, which bogs down the ring-buffer based control channel. We implemented the metadata exchange through a prepinned metadata segment (as discussed in the Eager scheme) for both protocols.

As shown in Figure 10, we see up to 5x speedup with Compact over Binary due entirely to the elimination of serialization overhead even when Compact has to transfer up to 5 times more data. However, we found that the time for deserialization of Binary was lower than the time required to fixup pointers for Compact.

The volume of data transfer is more in the zero-copy case as clients need to exchange metadata with the server. Furthermore, the size of each message is restricted by the maximum block size in the Compact. However at larger message sizes, we still expect to

\footnotetext{
${ }^{3}$ http://cci-forum.com/
} 


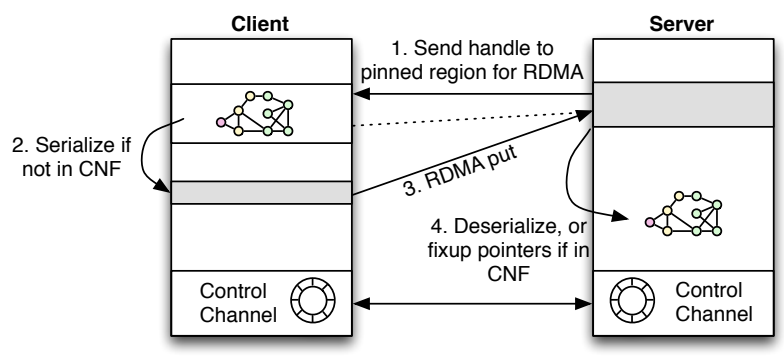

(a) Eager (push-based) RDMA protocol. Here the client wants to send a tree data structure to the server. This approach eliminates the initial rendezvous before communication at the expense of copying into a pre-pinned region on the server.

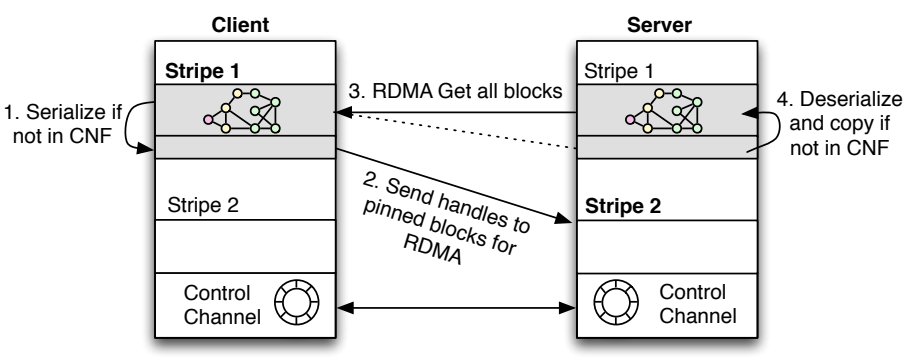

(b) Rendezvous (pull-based) RDMA protocol. This is the zero-copy case where the client sends metadata of the tree to the server. The server pulls data using remote read into a stripe that it has reserved for the client so that no pointer fixups are required.

Figure 9: The two RDMA data transfer schemes that were used for sending a tree from a client to the server.

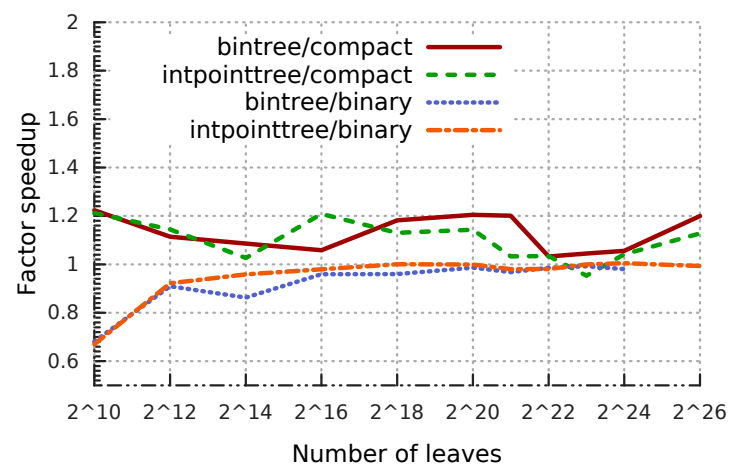

Figure 11: Factor speedup relative to the Eager (push-based) protocol discussed in Figure 9a that incurs a copy and/or pointer fixup overhead. Up to $20 \%$ speedup was observed for zero-copy rendezvous transfers with Compact trees.

Table 4: Requests handled by server for varying database sizes. The size corresponds to the space used by values in the Haskell heap.

\begin{tabular}{|c|c|c|c|}
\hline Keys & DB size & Binary & Compact \\
\hline 100 & $6.56 \mathrm{MB}$ & 17,081 & 69,570 \\
1,000 & $65.6 \mathrm{MB}$ & 15,771 & 63,285 \\
10,000 & $656 \mathrm{MB}$ & 15,295 & 57,008 \\
\hline
\end{tabular}

see performance improvements for zero-copy over the push variant for two reasons: first, an extra copy is eliminated and secondly, for large messages the cost of deserialization trumps the cost of additional data transfer. Figure 11 confirms our hypothesis. For Binary, we mostly see a slowdown except for tree depths above 25 as the cost of deserialization is never amortized by the additional communication penalty, whereas we see Compact do better at modest sizes as it avoids both copying and deserialization overheads. The communication throughput can further reduced for the zero-copy protocol by utilizing scatter/gather features available in modern Infiniband network hardware that provides vectorized data transfer between hosts.

\subsection{Case Study: In-Memory Key-Value Store}

We implemented a simple in-memory key-value store in Haskell, using compact regions to store values. Remote clients make re- quests upon the server to fetch the value at a given key. One possible implementation might store the table in a of map from Key ByteString, where the ByteString represents the serialized payload which should be sent in response. However, this is only workable if the server will only service fetch requests - the original values would be gone from memory, and could only be retrieved by deserializing the ByteString values. Of course, this deserialization is costly and doesn't support small, incremental updates to the values in question.

Alternatively, the implementor could choose to store Map Key Val directly, and opt to serialize on every fetch request. Leveraging lazy evaluation, it would be an elegant optimization to instead store Map Key ( $\mathrm{Val}$, ByteString), where the ByteString field is a thunk and is computed (and memoized) only if it is fetched by a client. Yet this option has its own drawbacks. The ByteString still needs to be recomputed in whole for any small modification of Val, and, further, the entire in-memory store now uses up to twice as much resident memory!

Using Compacts can improve both these problems, while keeping GC pressure low. If we store a Map Key (Compact Val) then (1) the value is ready to send at any point, (2) we are able to incrementally add to the compact without recreating it, and (3) the values are always in a "live" state where they support random access at any point within the Compact.

To test this approach, we built a TCP based server running on our evaluation platform. We evaluate this server in terms of client requests per second, using fixed-size values on each request (pointtree of depth 10, size 65.6KB). We use 16 client processes, each launching requests in a sequential loop, to saturate the server with as many requests as the $10 \mathrm{G}$ Ethernet network supports. In Table 4, we show how varying the size of the in-memory database changes request handling throughput, by changing the behavior of the memory system. Here we compare our Compact-based server against the Map Key Val solution, again using the Binary package for serializing Val, showing an increased throughput across a range of in-memory key-value store sizes.

\section{Related Work}

The idea of reusing an in-memory representation for network and disk communication originates from the very beginning of computing. It was common for old file formats to involve direct copies of in-memory data structures [24, 25, 29], in order to avoid spending CPU cycles serializing and deserializing in a resource constrained environment. More recently, libraries like Cap'n Proto [31] advo- 
cate in-memory representations as a general purpose binary network interchange format.

These applications and libraries are almost universally implemented in languages with manual memory management. However, there are some shared implementation considerations between these applications and compact normal forms. The literature on pointer swizzling [16, 32], for example, considers how pointers should be represented on disk. The idea of guaranteeing a structure is mapped to the same address occurs in many other systems. $[5,6,28]$

On the other hand, it is far less common to see this technique applied in garbage collected languages. One common mode of operation was to save the entire heap to disk in an image, so that it could be reloaded quickly; schemes like this were implemented in Chez Scheme and Smalltalk. Our goal was to allow manipulating subsets of the heap in question.

The one system we are aware of which organizes heap objects into regions in the same way is Expresso [9] for Java. Like our system, Expresso allocates heap objects into contiguous chunks of memory which can then be transmitted to other nodes. However, while there are similarities in the underlying implementations, our API is substantially safer: Expresso is implemented in a language which supports mutation on all objects, which means that there is no invariant guaranteeing that all pointers are internal to a compact block. Compact Normal Forms do have this invariant, which means we can optimize garbage collection and avoid dangling pointers. Other systems [7] send the literal format but don't try to maintain a contiguous representation; thus a traversal is still necessary as part of the serialization step.

Message passing in distributed computation There is a lot of prior work in systems for distributed computation. The Message Passing Interface (MPI) is the standard for message communication in many languages, and emphasizes avoiding copying data structures. However, MPI assumes that data lives in a contiguous buffer prior to sending: it is up to the high-level language to arrange for this to be the case.

Message passing implementations in high-level languages like Java and Haskell are more likely to have a serialization step. Accordingly, there has been some investigation on how to make this serialization fast: Java RMI [27], for example, improved serialization overhead by optimizing the serialization format in question; Scala Pickler [26] approaches the problem by statically generating code to serialize each data structure in question. However, except in the cases mentioned in the previous section, most serialization in these languages doesn't manage to achieve zero-copy.

It is worth comparing our API to existing message passing APIs in distributed Haskell systems. Our approach is more in keeping with previous systems like Eden [3], where the system offers builtin support for serializing fully evaluated, non-closure data. Cloud Haskell [10], on the other hand attempts to support the transmission of higher-level functions with a combination of extra language techniques. Like Cloud Haskell, our system works best if identical Haskell code is distributed to all nodes, although we can accommodate (with performance loss) differing executables.

Regions and garbage collection It is folklore [4] that in the absence of mutable data, generational garbage collection is very simple, as no mutable set must be maintained in order that a backwards pointer from the old generation to the new generation is handled properly. In this sense, a compact region is simply a generalization of generational garbage collection to have arbitrarily many tenured generations which are completely self-contained. This scheme bears similarity to distributed heaps such as that in Singularity [14], where each process has a local heap that can be garbage collected individually. Of course, the behavior of data in a compact region is much simpler than that of a general purpose heap.

The idea of collecting related data into regions of the heap has been explored in various systems, usually in order to improve data locality. $[1,8,11]$ At the static end of the spectrum, region systems $[12,30]$ seek to organize dynamically allocated data into regions which can be freed based on static information, eliminating the need for a tracing garbage collector. MLKit [13] combines region inference with tracing garbage collection; their garbage collection algorithm for regions bears some similarities to ours; however, since we don't trace data living in a region, our algorithm is simpler at the cost of space wastage for objects in a compact region which become dead-a tradeoff which is also familiar to region systems.

\section{Conclusions}

In programming languages, abstraction is naturally sometimes at odds with performance, especially with regards to garbage collection versus manual memory management. In this paper, we have tried to show how compact regions can be a semantically simple primitive that still brings good performance benefits to the table. We believe this sort of region management may prove to be a practical compromise for managing heap layout, just as semi-explicit parallelism annotations have proven a useful compromise.

\section{Acknowledgments}

Edward Z. Yang is supported by the DoD through the NDSEG. Support for this work was also provided by NSF grant CCF1218375 and CCF-1453508.

\section{References}

[1] E. D. Berger, B. G. Zorn, and K. S. McKinley. Reconsidering custom memory allocation. ACM SIGPLAN Notices, 37:1, 2002.

[2] R. L. Bocchino Jr, V. S. Adve, D. Dig, S. V. Adve, S. Heumann, R. Komuravelli, J. Overbey, P. Simmons, H. Sung, and M. Vakilian. A type and effect system for deterministic parallel Java. ACM Sigplan Notices, 44(10):97-116, 2009.

[3] S. Breitinger, U. Klusik, and R. Loogen. From (sequential) Haskell to (parallel) Eden: An Implementation Point of View. Symposium on Programming Language Implementation and Logic Programming - PLILP, pages 318-334, 1998.

[4] L. Cardelli. The Functional Abstract Machine. Technical Report TR107, AT\&T Bell Laboratories, 1983.

[5] J. Chase, F. Amador, E. Lazowska, H. Levy, and R. Littlefield. The Amber system: parallel programming on a network of multiprocessors. ACM SIGOPS Operating Systems Review, 23(December 1989):147-158, 1989.

[6] J. S. Chase, H. M. Levy, E. D. Lazowska, and M. Baker-Harvey. Lightweight shared objects in a 64-bit operating system. In $A C M$ SIGPLAN Notices, volume 27, pages 397-413, 1992.

[7] S. Chaumette, P. Grange, B. Métrot, and P. Vignéras. Implementing a High Performance Object Transfer Mechanism over JikesRVM. 2004.

[8] T. M. Chilimbi and J. R. Larus. Using generational garbage collection to implement cache-conscious data placement. In ACM SIGPLAN Notices, volume 34, pages 37-48, 1999.

[9] L. Courtrai, Y. Maheo, and F. Raimbault. Expresso: a Library for Fast Java Objects Transfer. In Myrinet User Group Conference (MUG), 2000.

[10] J. Epstein, A. P. Black, and S. Peyton-Jones. Towards Haskell in the cloud. ACM SIGPLAN Notices, 46(Section 4):118, 2012.

[11] T. D. S. Gene Novark. Custom Object Layout for Garbage-Collected Languages. Techreport, 2006.

[12] D. Grossman, G. Morrisett, T. Jim, M. Hicks, Y. Wang, and J. Cheney. Region-based memory management in Cyclone. In PLDI, 2002. 
[13] N. Hallenberg, M. Elsman, and M. Tofte. Combining region inference and garbage collection. In PLDI, volume 37, page 141, May 2002.

[14] G. C. Hunt and J. R. Larus. Singularity: rethinking the software stack. SIGOPS OSR, 2007.

[15] S. L. P. Jones. Implementing lazy functional languages on stock hardware: the Spineless Tagless G-machine. Journal of Functional Programming, 2:127-202, 1992.

[16] A. Kemper and D. Kossmann. Adaptable pointer swizzling strategies in object bases: design, realization, and quantitative analysis. The VLDB Journal, 4:519-566, 1995.

[17] R. Lämmel and S. P. Jones. Scrap your boilerplate with class: Extensible generic functions. SIGPLAN Not., 40(9):204-215, Sept. 2005.

[18] J. Launchbury. A natural semantics for lazy evaluation. In Proceedings of the 20th ACM SIGPLAN-SIGACT symposium on Principles of programming languages, pages 144-154. ACM Press, 1993.

[19] R. Loogen, Y. Ortega-mallén, and R. Peña marí. Parallel functional programming in Eden. Journal of Functional Programming, 15(3):431-475, May 2005.

[20] P. Maier and P. Trinder. Implementing a high-level distributedmemory parallel haskell in haskell. In Implementation and Application of Functional Languages, pages 35-50. Springer, 2012.

[21] S. Marlow, T. Harris, R. P. James, and S. Peyton Jones. Parallel generational-copying garbage collection with a block-structured heap. In Proceedings of the 7th international symposium on Memory management, ISMM '08, pages 11-20, New York, NY, USA, 2008. ACM.

[22] K. McKelvey and F. Menczer. Design and prototyping of a social media observatory. In Proceedings of the 22nd international conference on World Wide Web companion, WWW'13 Companion, pages 13511358, 2013.

[23] K. McKelvey and F. Menczer. Truthy: Enabling the Study of Online Social Networks. In Proc. 16th ACM Conference on Computer Sup- ported Cooperative Work and Social Computing Companion (CSCW), 2013.

[24] Microsoft Corporation. Word (. doc) Binary File Format. https://msdn.microsoft.com/en-us/library/office/ cc313153 (v=office.12) . aspx, 2014.

[25] Microsoft Corporation. Bitmap Storage. https://msdn. microsoft. com/en-us/library/dd183391. aspx, 2015.

[26] H. Miller, P. Haller, E. Burmako, and M. Odersky. Instant pickles: Generating object-oriented pickler combinators for fast and extensible serialization. In Proceedings of the 2013 ACM SIGPLAN International Conference on Object Oriented Programming Systems Languages \&\#38; Applications, OOPSLA '13, pages 183-202, New York, NY, USA, 2013. ACM.

[27] C. Nester, M. Philippsen, and B. Haumacher. A more efficient RMI for Java. In Proceedings of the ACM 1999 conference on Java Grande, pages 152-159. ACM, 1999.

[28] E. Shekita and M. Zwilling. Cricket: A Mapped, Persistent Object Store. Center for Parallel Optimization, Computer Sciences Department, University of Wisconsin, 1996.

[29] TIS Committee. Tool Interface Standard (TIS) Executable and Linking Format (ELF) Specification (Version 1.2). Technical Report May, 1995.

[30] M. Tofte, L. Birkedal, M. Elsman, and N. Hallenberg. A retrospective on region-based memory management. Higher-Order and Symbolic Computation, 17(3):245-265, 2004.

[31] K. Varda. Cap'n Proto. https://capnproto.org/, 2015.

[32] P. Wilson and S. Kakkad. Pointer swizzling at page fault time: efficiently and compatibly supporting huge address spaces on standard hardware. [1992] Proceedings of the Second International Workshop on Object Orientation in Operating Systems, 1992. 